\title{
Transient pain and paresthesias in the hand-ulnar neuropathy secondary to compression from a low-lying medial triceps muscle and tendon insertion
}

\author{
Luis S. Beltran • Oren Lerman • Sheel Sharma • \\ Jenny T. Bencardino
}

Published online: 14 June 2013

(C) ISS 2013

\section{Part II}

Diagnosis

Ulnar neuropathy secondary to compression from a lowlying medial triceps muscle and tendon insertion

\section{Discussion}

Ulnar nerve dislocation and snapping triceps syndrome can cause compressive ulnar neuropathy at the elbow [1] during elbow flexion. The ulnar nerve may dislocate in the presence or absence of snapping triceps syndrome and the two conditions are clinically indistinguishable, therefore, imaging is important for diagnosis [2]. Isolated ulnar nerve dislocation without snapping triceps syndrome can be treated operatively by cubital tunnel release or medial humeral epicondylectomy and ulnar nerve transposition [3]. Operative treatment of snapping triceps syndrome includes excision of the dislocating portion of the medial triceps muscle in addition

The case presentation can be found at doi: 10.1007/s00256-013-1661-0

L. S. Beltran $(\bowtie)$

Department of Radiology, NYU Hospital for Joint Diseases,

301 East 17th Street, New York, NY 10003, USA

e-mail: luis.beltran@nyumc.org

O. Lerman

Department of Plastic Surgery, Lenox Hill Hospital,

New York, NY, USA

S. Sharma

Department of Plastic Surgery, NYU Plastic Surgery Associates, 301 East 17th Street, New York, NY 10003, USA

J. T. Bencardino

NYU Hospital for Joint Diseases, 301 East 17th Street,

New York, NY 10003, USA to ulnar nerve decompression and transposition [2]. Failure to recognize that dislocation of both the medial triceps muscle and ulnar nerve can exist concurrently may result in persistent snapping, elbow pain, and even ulnar nerve symptoms after a technically successful ulnar nerve transposition [4].

Dislocation of the ulnar nerve from the cubital tunnel has been reported in approximately $16 \%$ of healthy subjects. A proposed cause of ulnar nerve dislocation is congenital absence of the cubital tunnel retinaculum [5]. Snapping triceps syndrome was first described in 1970 by Rolfsen. The prevalence and exact etiology of this syndrome are unknown [2]. Symptoms include medial elbow pain, snapping sensation with elbow flexion, and/or ulnar neuropathy [2]. Acquired causes include increased bulk of the triceps muscle such as with bodybuilding and overhead activities such as pitching and weightlifting [2]. Congenital causes include accessory triceps muscle slips and prominence of the medial triceps muscle and/or separate insertion of the medial triceps, which may cause ulnar nerve instability and secondary neuritis [2]. Osseous abnormalities such as fracture or developmental cubitus varus deformity have also been associated with dislocation of the triceps, dislocation of the ulnar nerve, and ulnar neuropathy $[2,6]$.

A low-lying insertion of the medial triceps muscle is likely a developmental variant that may predispose the ulnar nerve to become compressed or the ulnar nerve and/or the medial triceps muscle to become dislocated resulting in ulnar neuropathy in certain cases of overuse [7] by causing mass effect and crowding of the ulnar nerve in the cubital tunnel during elbow flexion. The diagnosis in our case is supported by MR findings with surgical correlation (Fig. 1) and by postoperative outcome with complete relief of symptoms. Interestingly, our patient also had a low-lying medial triceps muscle insertion in the asymptomatic contralateral right elbow (Fig. 2), but this did not seem to be associated with ulnar neuropathy.

Determining the correct site and cause of compression of the ulnar nerve can be challenging. Although electrodiagnostic studies are the gold standard, patient discomfort and inconclusive 
Fig. 1 Ulnar neuritis and lowlying medial triceps. Axial proton density and $\mathrm{T} 2$ fat-suppressed MR images of the left elbow in the patient presented in the testyourself question. There is enlargement and increased T2 signal of the ulnar nerve (solid arrow) at the cubital tunnel and a low-lying medial triceps muscle (dashed arrow) and tendon (curved arrow)

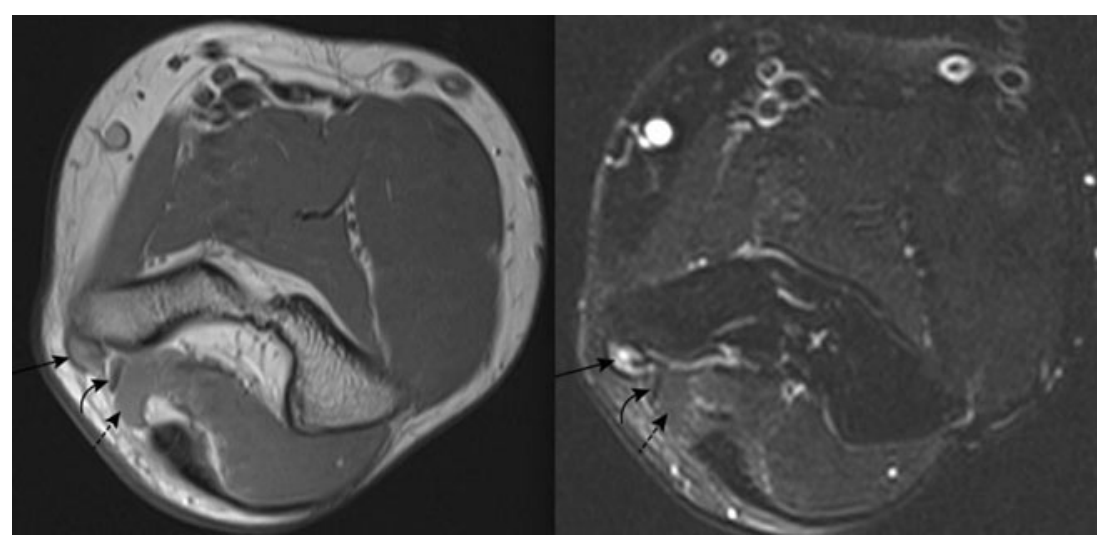

Fig. 2 Low-lying medial triceps in asymptomatic elbow. Axial proton density and $\mathrm{T} 2$ fatsuppressed MR images of the right elbow in the same patient presented in the test-yourself question. There is no significant enlargement and increased T2 signal of the ulnar nerve (solid arrow) at the cubital tunnel despite having a low-lying medial triceps muscle (dashed arrow) and tendon (curved arrow)

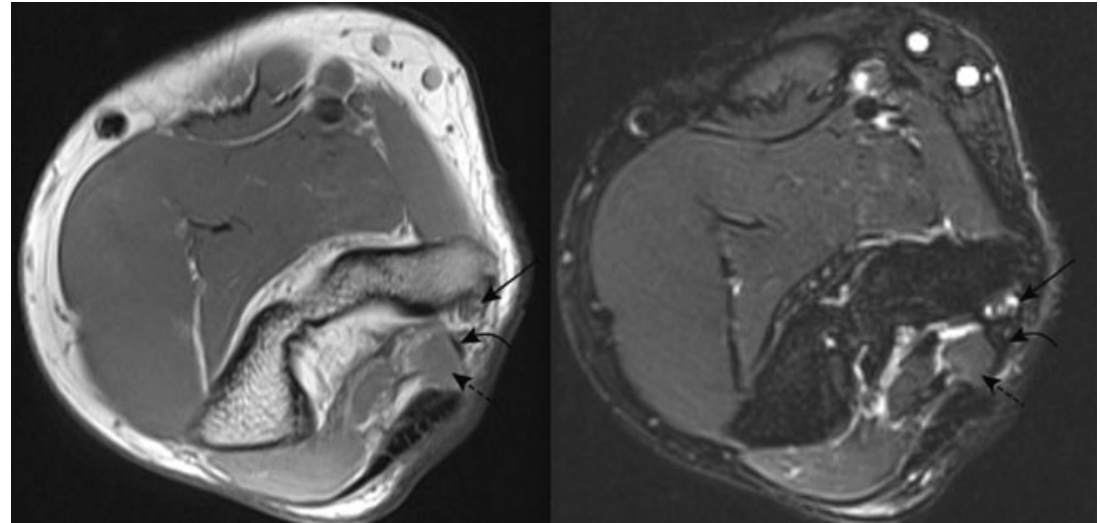

results in up to one-third of cases have been reported [8]. MRI and/or ultrasound can confirm the clinical diagnosis and identify the site and cause of nerve compression. On T1-weighted images, thickening of the nerve with enlargement and/or effacement of nerve fascicles are characteristic [9]. On T2W images, abnormal nerves show hyperintense signal, approaching that of adjacent vessels. Denervation muscle changes may also be seen including muscle edema and/or fat atrophy. Dynamic ultrasound can differentiate isolated ulnar nerve dislocation from snapping triceps syndrome [1]. In isolated ulnar nerve dislocation, the ulnar nerve will dislocate anterior to the medial epicondyle with elbow flexion and the medial triceps muscle will remain posterior to the medial epicondyle. In contrast, in snapping triceps syndrome, both the ulnar nerve and the medial triceps muscle will dislocate anterior to the medial epicondyle (Fig. 3) [1]. The abnormal nerve will also demonstrate thickening and loss

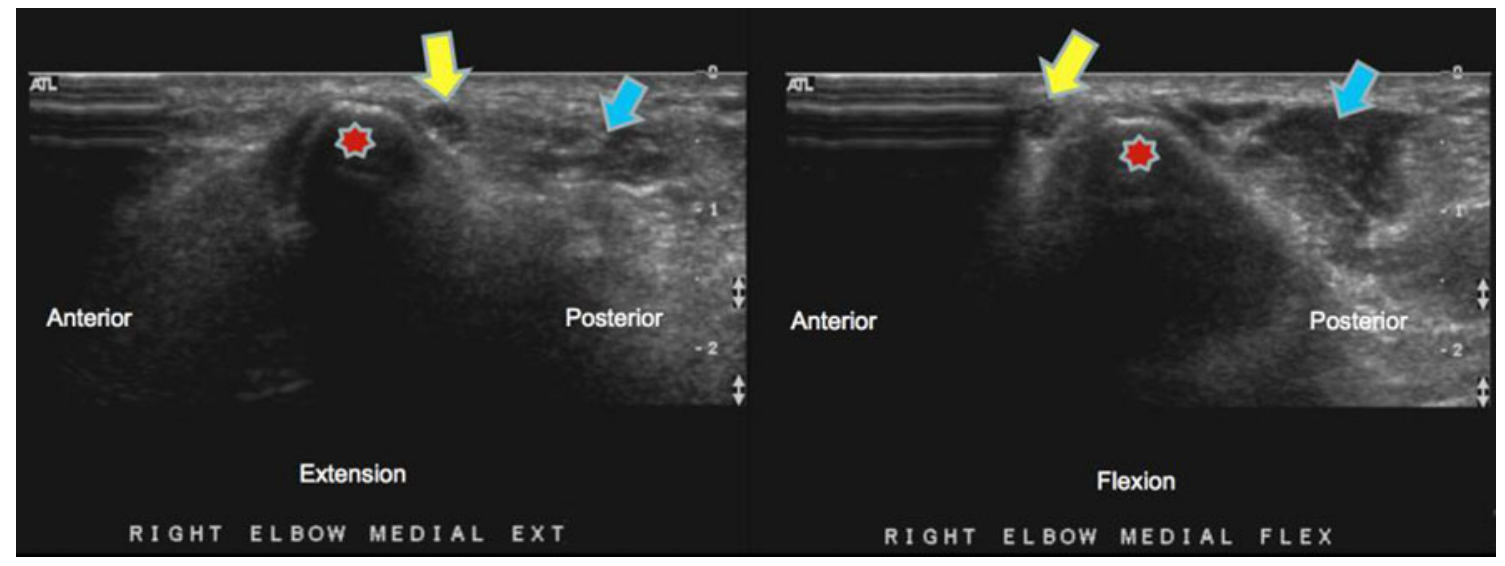

Fig. 3 Ulnar nerve dislocation associated with a low-lying medial triceps muscle: 31-year-old male with clinical symptoms of snapping sensation in the right elbow with flexion and ulnar-sided paresthesias in the hand. Transaxial dynamic ultrasound of the cubital tunnel at the medial aspect of the elbow shows dislocation of the ulnar nerve (yellow arrow) from the cubital tunnel, anterior to the medial humeral epicondyle (red asterisk) on the flexion image, secondary to mass effect from a low-lying medial triceps muscle (blue arrow) 
of the normal honeycomb echotexture becoming homogeneously hypoechoic [10].

The differential diagnosis in this case includes spaceoccupying lesions and congenitally or acquired osseous abnormalities causing compression of the ulnar nerve, and the presence of an anconeus epitrochlearis muscle.

In conclusion, a low-lying medial triceps muscle and tendon insertion should be considered in the differential diagnosis of ulnar neuritis and dislocation.

Disclosure The authors declare that they have no conflicts of interest.

\section{References}

1. Jacobson JA, Jebson PJ, Jeffers AW, Fessell DP, Hayes CW. Ulnar nerve dislocation and snapping triceps syndrome: diagnosis with dynamic sonography-report of three cases. Radiology. 2001;220(3):601-5.

2. Spinner RJ, Goldner RD. Snapping of the medial head of the triceps and recurrent dislocation of the ulnar nerve. Anatomical and dynamic factors. J Bone Joint Surg Am. 1998;80(2):239-47.
3. Kroonen LT. Cubital tunnel syndrome. Orthop Clin N Am. 2012;43(4):475-86.

4. Spinner RJ, O'Driscoll SW, Jupiter JB, Goldner RD. Unrecognized dislocation of the medial portion of the triceps: another cause of failed ulnar nerve transposition. J Neurosurg. 2000;92(1): $52-7$.

5. O'Driscoll SW, Horii E, Carmichael SW, Morrey BF. The cubital tunnel and ulnar neuropathy. J Bone Joint Surg Br. 1991;73(4): $613-7$.

6. Spinner RJ, O'Driscoll SW, Davids JR, Goldner RD. Cubitus varus associated with dislocation of both the medial portion of the triceps and the ulnar nerve. J Hand Surg Am. 1999;24(4):718-26.

7. Beltran LS, Sussmann A, Bencardino JT, Rosenberg ZS, Beltran J, Jazrawi L, et al. Low insertion of the medial head of triceps muscle at the elbow: normal anatomy, variants and pathology based on cadaveric and MR studies. Skeletal Radiol. 2012;41(6):757-8.

8. Dellon ALA. Management of peripheral nerve problems in the upper and lower extremity using quantitative sensory testing. Hand Clin. 1999;15(4):697-x.

9. Chhabra A, Lee PP, Bizzell C, Soldatos T. 3 Tesla MR neurographytechnique, interpretation, and pitfalls. Skeletal Radiol. 2011;40(10): 1249-60.

10. Kara M, Ozçakar L, De Muynck M, Tok F, Vanderstraeten G. Musculoskeletal ultrasound for peripheral nerve lesions. Eur J Phys Rehabil Med. 2012;48(4):665-74. 